\title{
Does granulocyte-colony stimulating factor stimulate peripheral nerve regeneration? An experimental study on traumatic lesion of the sciatic nerve in rats
}

\author{
Doerthe Keiner $^{1 \star}$, Harald von Pein ${ }^{2 \star}$, Jacek Szczygielski ${ }^{1,3}$, Andreas Kramer ${ }^{4}$, Axel Heimann ${ }^{5}$, \\ Oliver Kempski ${ }^{5}$, Clemens Sommer ${ }^{2}$, Joachim Oertel ${ }^{1}$
}

\begin{abstract}
${ }^{1}$ Department of Neurosurgery, Saarland University Medical Centre and Saarland University Faculty of Medicine, Homburg/Saar, Germany ${ }^{2}$ Division of Neuropathology, University Medical Centre of the Johannes-Gutenberg-University, Mainz, Germany

${ }^{3}$ Institute of Medical Sciences, University of Rzeszow, Poland

${ }^{4}$ Department of Neurosurgery, University Medical Centre of the Johannes-Gutenberg-University, Mainz, Germany ${ }^{5}$ Institute of Neurosurgical Pathophysiology, University Medical Centre of the Johannes-Gutenberg-University, Mainz, Germany
\end{abstract}

\begin{abstract}
Aim of the study. To analyse the therapeutic potential of granulocyte-colony stimulating factor (G-CSF) treatment using a rat model of traumatic sciatic nerve lesion.

Clinical rationale for the study. G-CSF has proven strong neurotrophic properties in various models of ischaemic and traumatic brain injury. Fewer studies exist regarding the influence of G-CSF on posttraumatic peripheral nerve regeneration. Currently, the possibilities of pharmacological prevention or treatment of mechanical nerve injury are limited, and there is an urgent need to find new treatment strategies applicable in clinical situations.

Materials and methods. A controlled traumatic right sciatic nerve lesion was set using a waterjet device. Three treatment groups were created. In the first group, G-CSF was administered after sciatic nerve injury. The second group received G-CSF before and after trauma, while the third group was treated with glucose 5\%-solution. Sciatic nerve function was assessed clinically and electrophysiologically at day 1, and after weeks 1, 2, 4 and 6 . Additionally, a-motoneurons of the spinal cord and sciatic nerve fibres were counted at week 6.

Results. Clinically, rats in both G-CSF groups improved faster compared to the control group. Additionally, animals treated with G-CSF had a significantly better improvement of motor potential amplitude and motor nerve conduction velocity at week 6 ( $p$ $<0.05$ ). Histologically, G-CSF treatment resulted in a significantly higher number of a-motoneurons and small myelinated nerve fibres compared to placebo treatment $(p<0.05)$.

Conclusions and clinical implications. Under G-CSF treatment, the recovery of motor nerve conduction velocity and amplitude was enhanced. Further, signs of nerve regeneration and preservation of a-motoneurons were observed. These results indicate that G-CSF might accelerate and intensify the recovery of injured nerves. Thus, treatment with G-CSF may be beneficial for patients with peripheral nerve damage, and should be explored in further clinical studies.
\end{abstract}

Key words: G-CSF, nerve regeneration, peripheral nerve lesion, traumatic nerve injury, waterjet dissection (Neurol Neurochir Pol 2021; 55 (5): 469-478)

Address for correspondence: Joachim Oertel, Department of Neurosurgery, Saarland University Medical Centre, Kirrberger Str. 90.5, 66421 Homburg-Saar, Germany; e-mail: joachim.oertel@uks.eu

Received: 11.11.2020 Accepted: 12.07.2021 Early publication date: 19.10.2021

This article is available in open access under Creative Common Attribution-Non-Commercial-No Derivatives 4.0 International (CC BY-NC-ND 4.0) license, allowing to download articles and share them with others as long as they credit the authors and the publisher, but without permission to change them in any way or use them commercially.

${ }^{*}$ D. Keiner and H. v. Pein contributed equally to this work 


\section{Introduction}

Today's neurotrauma research focuses on injury of the central nervous system (CNS). However, one should not forget that peripheral nerve injury as a subject of neurotrauma studies deserves the same attention as do brain or spinal cord trauma, since injury of peripheral or cranial nerves can also result in permanent functional loss of differing degrees of severity, and therefore the potential of neuroprotective treatment should also be explored for the peripheral part of the nervous system. In the past, different agents with possible neurotrophic properties have been investigated in detail. Recently, the use of granulocyte-colony stimulating factor (G-CSF) has been reported as a very promising treatment strategy $[1,2]$. This induces proliferation and mobilisation of haematopoietic cells. Further, it regulates maturation and survival of neutrophil granulocyte precursors $[1,2]$. The stimulation of neutrophil granulocyte precursors is commonly applied in the management of chemotherapy-associated neutropenia or in stem-cell transplantation [3,4]. Several studies involving different in vitro and in vivo experimental stroke models have proven that G-CSF induces neuroprotective and neuroregenerative properties $[1,2,5-7]$. This has also been reported in human subjects with acute stroke $[3,8]$. Besides its anti-apoptotic effect, G-CSF enhances angiogenesis after ischaemia and promotes neurogenesis $[1,2]$. Furthermore, it has been shown that G-CSF improves the recovery of sensorimotor, as well as cognitive, functions after ischaemia [2].

In the peripheral nervous system (PNS), it has recently been observed that G-CSF protects $\alpha$-motoneurons of the spinal cord from apoptosis after axotomy of sciatic nerves in chimeric mice [4]. Furthermore, in an experimental mouse model of amyotrophic lateral sclerosis (AML), G-CSF increased the survival of motoneurons in vivo and in vitro and decreased muscular nerve denervation and atrophy in SOD1 (G93A) transgenic mice $[9,10]$. However, the neuroprotective or neuroregenerative properties of G-CSF in traumatic peripheral nerve injury have not been fully evaluated.

The recently developed model of waterjet-induced injury of the sciatic nerve creates a good opportunity to analyse this potential. By adapting a surgical device for waterjet dissection [11-18], one may achieve injury to the nerve wherein the degree of damage can be precisely regulated. Based on these principles, a rat model of waterjet-induced injury to vestibulocochlear and sciatic nerves has been established $[19,20]$. According to the previous results, the reliability and replicability of this experimental paradigm is comparable with the canonical model of sciatic nerve crush [4, 21-23]. Moreover, waterjet nerve injury models enable the analysis of functional nerve recovery after a partial lesion with damaged microstructure while maintaining continuity, thus simulating the common clinical situation of iatrogenic nerve injury during a surgical procedure $[19,20]$.
This current study was prompted by a desire to analyse the impact of G-CSF on peripheral nerve recovery after a moderate trauma (defined as significant and prolonged damage but with recovery capability). We investigated both iatrogenic and incidental injury scenarios, with G-CSF administration before and after trauma, and a waterjet-based model of traumatic nerve injury.

\section{Clinical rationale for the study}

There is a growing need to expand the concept of neuroprotective therapies from brain and spinal cord research into the realm of clinical studies on peripheral nerve injury. The main impetus is the growing number of cases resulting from the long list of possible causes of nerve damage. Firstly, accidental injury to the limbs as encountered in road accidents, gunshot wounds or sports injuries may lead to severe nerve damage [24,25]. Further, a nerve injury can result from surgical procedures. For example, simple manipulation of cranial nerves during surgery at the skull base often causes functional impairment, even in macroscopically intact nerves. Additionally, limb surgery, or even an inappropriate positioning on the surgical table, can lead to nerve compression and subsequently to its functional and structural damage $[26,27]$. For surgery-related nerve injury, the timing of a trauma can be predicted. Thus, a potential preventive treatment would be possible.

However, regardless of the cause of nerve injury (accidental or iatrogenic), the grade of the recovery and its duration cannot be prognosticated in every case and there is still a vast population of patients where nerve regeneration is prolonged, arrested, or simply incomplete. Thus, any treatment method able to accelerate or enable recovery in peripheral nerve damage is of paramount importance for clinical practice. Our current study represents an attempt to test one of the promising therapeutic strategies under animal experiment conditions.

\section{Materials and methods}

\section{Study design}

This study was approved by the local Ethics Committee and by the institutional Animal Welfare Representative. Male adult Sprague-Dawley rats weighting 300-400g were used in this study. Animals were kept under controlled light conditions with a $12 \mathrm{~h} / 12 \mathrm{~h}$ light/dark cycle. Food and water were provided ad libitum. The following experimental groups $(\mathrm{n}=24$ each group) were created: in all groups, traumatic injury to the right sciatic nerve was applied during the surgical procedure, as described below. Twenty-four animals were treated with recombinant human G-CSF (Neupogen", filgrastim, Amgen $\mathrm{GmbH}$, Munich, Germany) administered intravenously (i.v.) at a dose of $60 \mu \mathrm{g} / \mathrm{KGBW}$ on days 1,3 and 5 after surgery (Group 1). To address the question, if an additional administration of G-CSF prior to a planned surgery with anticipated traumatic impact on a peripheral nerve (i.e. tumour surgery or in severe nerve compression) might result in a better nerve regeneration 
compared to G-CSF after surgery, and may become a prophylactic measure in a planned surgical procedure, in 24 animals a G-CSF dose of $60 \mu \mathrm{g} / \mathrm{KGBW}$ was given i.v. one day prior to surgery and at days 1, 3 and 5 after surgery (Group 2). In the remaining 24 animals, 5\%-glucose (G5\%) - solution was administered i.v. as a placebo (Group 3).

Twelve animals in each group were sacrificed for histological evaluation of the sciatic nerve after week 1 . The remaining 12 animals were sacrificed after six weeks for histological evaluation of the sciatic nerve and its spinal cord section.

The neurological function was assessed using pace analysis according to the sciatic functional index (SFI) after $24 \mathrm{hrs}$ and after 1, 2, 4 and 6 weeks. Additional instrumental analysis of the motor nerve conduction velocity (NCV) and the motor potential amplitude was performed prior to nerve injury and at the end of the surgery after wound closure, and repeated $24 \mathrm{~h}$ and 1, 2, 4 and 6 weeks after injury (Nicolet Viking ${ }^{\circledR}$ and Medelec $^{\text {mw }}$ Synergy N-EP - EMG/EP monitoring system, Version 12.2, Natus Neurology Inc, Planegg, Germany).

\section{Scoring of neurological deficits}

All rats underwent pre- and postoperative walking track analysis in a confined walkway. Hind paw prints were recorded using black ink. The factors for SFI were calculated as described by De Medinaceli et al [28]. Additionally, the number of steps per metre and possible limping following nerve lesion was evaluated as previously described [20]. An SFI of 0 to -5 was considered to be 'intact motor function', an SFI of -6 to -50 was considered to be 'marked neurological deficit', and an SFI of -51 to -100 was considered to be 'severe neurological deficit'.

\section{Surgical and lesion procedure}

The rats were anaesthetised with chloralhydrate solution intraperitoneally (i.p.) at a dose of $36 \mathrm{mg} / \mathrm{kg}$ body weight (BW) before surgery. Perioperative analgesia was performed with tramadol i.p. at a dose of $50 \mathrm{mg} / \mathrm{kg}$ BW. A posterior-laterally skin incision was done parallel to the right femur and the muscle fascia of the gluteus muscles was opened. The sciatic nerve was carefully exposed at midthigh level with the aid of a wound expander. Under microscopic view, the nerve was mobilised from the surrounding muscle fascia until it was exposed from the sciatic notch exit to the division of motor branches. After preparation, the rat was placed on a computer-controlled linear device for dissection of the sciatic nerve (Software Servomanager 6.4.1, Parker Automation; Erbe Elektromedizin Company, Fig. 1). After dissection, the muscle fascia and the skin was closed with 4-0 sutures.

For sciatic nerve dissection, an Erbejet 2 (Erbe Elektromedizin Company, Tuebingen, Germany) was used. A waterjet is generated via a medium converter with an electronically-controlled mechanical system (double piston pump) with a pressure ranging from 1 to $80 \mathrm{bar}$. The medium converter is connected to a pencil-like handpiece consisting of a narrow

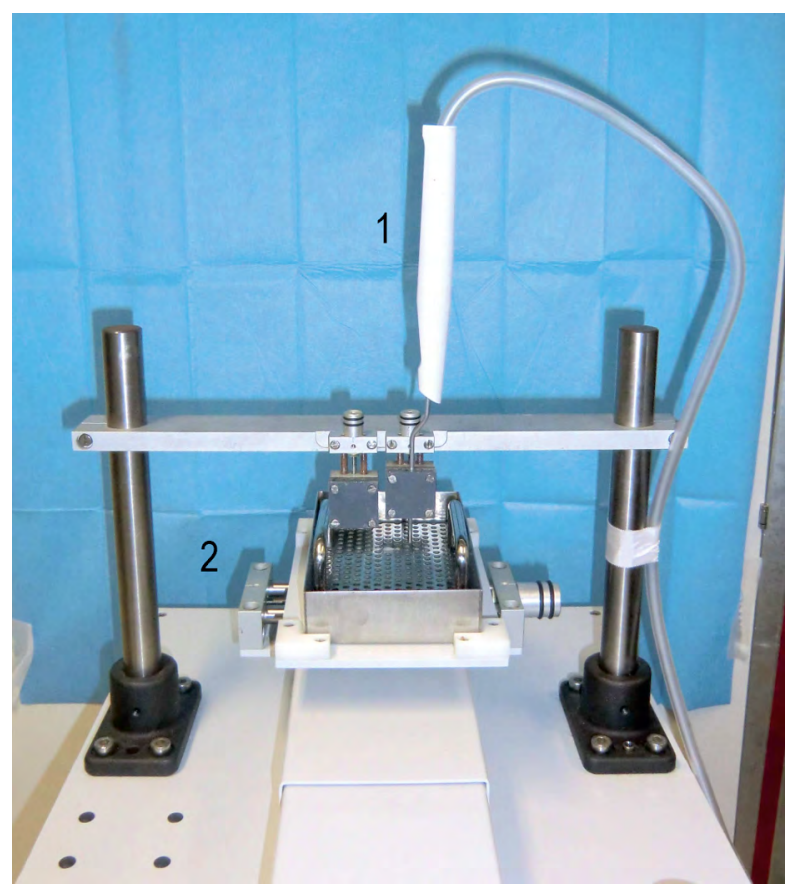

Figure 1. Computer-controlled linear device (Software Servomanager 6.4.1, Parker Automation; Erbe Elektromedizin Company) for application of waterjet dissection. Bayonet-shaped waterjet applicator (1) is placed above platform for animals with movement control (2)

nozzle (diameter of $120 \mu \mathrm{m}$ ), and a surrounding suction tube. The generated water jet is a non-rotatory thin lamina of liquid. Sterile $0.9 \%$ isotonic saline is emitted as separating medium with a volume flow of $1-55 \mathrm{~mL} / \mathrm{min}$. The pressure can be adjusted. Depending on the surgical procedure, several different settings can be selected. This system has been approved by the regulatory authorities in Germany and the United States of America for surgical use in humans.

To obtain a marked sciatic nerve lesion with retained nerve continuity, the jet intensity was set for 50 bar (Fig. 2A, B). It was applied at a 90-degree angle and with a cutting distance of $2 \mathrm{~mm}$ from the nozzle's tip to the nerve surface.

\section{Histological examination}

Histological analysis of the sciatic nerves, as well as counting of a-motoneurons, was performed by blinded investigators. The sciatic nerves were fixed in glutaraldehyde (3\% glutaraldehyde in $0.1 \mathrm{~mol} / \mathrm{L}$ sodium cacodylate buffer, cooled to $4^{\circ} \mathrm{C}$ ) and embedded in epon resin. Semi-thin sections of the dissection area were stained with methylene blue and analysed by light microscope (Olympus BH2, Hamburg, Germany) for signs of direct sciatic nerve injury and nerve regeneration. For morphometric analysis of nerve-regeneration, the entire nerve cross-section was photographed. Nerve fibre diameters and nerve fibres $/ \mathrm{mm}^{2}$ were analysed in two representative areas with an edge length of $0.1 \mathrm{~mm}$ of each case. Spinal cords were removed in toto and fixed in $4 \%$ 


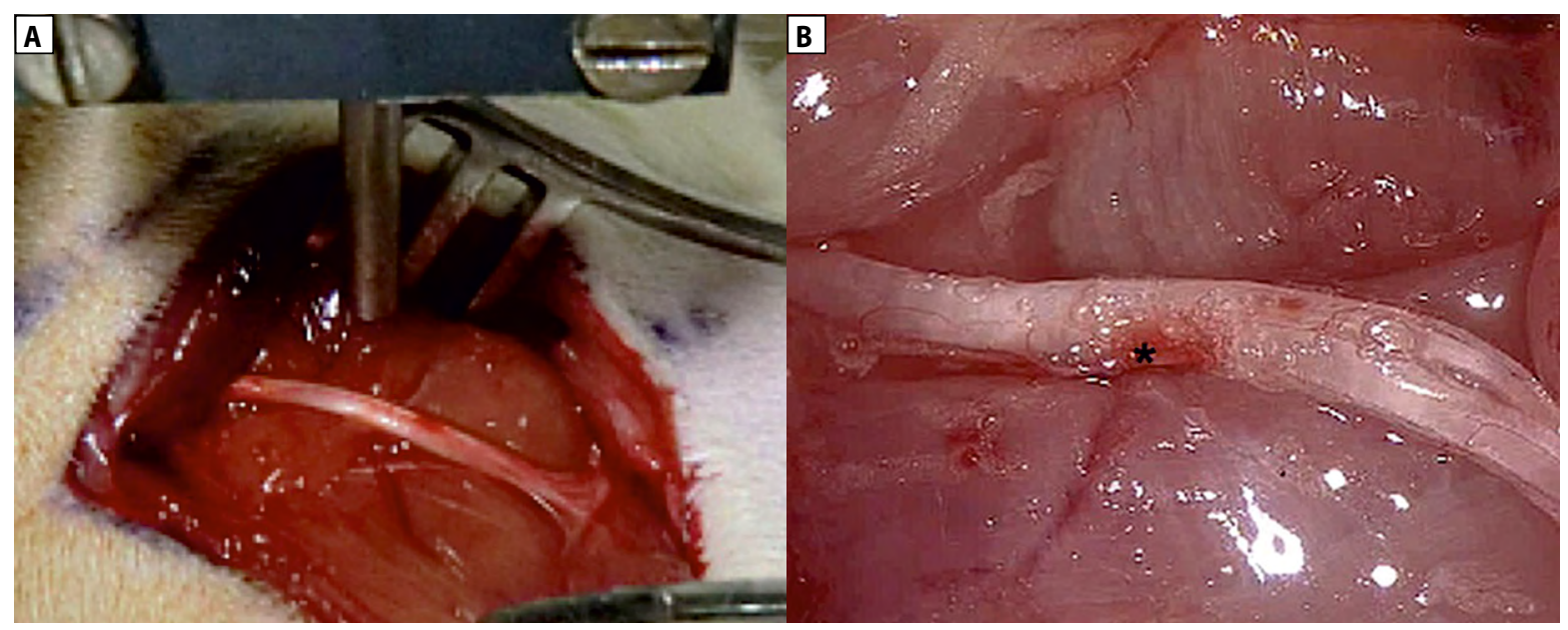

Figure 2. Microscopic view of right sciatic nerve. Setting of 50 bar lesion (A) and microscopic view of sciatic nerve directly after applied lesion (B)

paraformaldehyde. Thereafter, the relevant lumbar parts of the spinal cord were paraffin-embedded and cut into $10 \mu \mathrm{m}$ thick slices for counting of $\alpha$-motoneurons. For every spinal cord, 10 slices were analysed. Nissl and haematoxylin and eosin staining were performed. All neurons in laminas 8 and 9 of the ventral horn that were clearly identifiable in the staining and were $\geq 300 \mu \mathrm{m}^{2}$ in size were counted as $\alpha$-motoneurons (SIS AnalySIS, Olympus).

\section{Statistics}

For statistical evaluation of SFI and electrophysiological examinations, one- and two-way analysis of variance (ANOVA) was calculated with GraphPad Prism. A p value of less than 0.05 was considered statistically significant. For statistical analysis of the SFI, post-injury worsening of the SFI was divided, as described above, into 'intact motor function' $=1(0$ to -5$)$, 'marked neurological deficit' $=2(-6$ to -50$)$, or 'severe neurological deficit' $=3(-51$ to -100$)$.

For statistical evaluation of nerve fibres and $\alpha$-motoneurons, the total number of the sciatic nerve's fibres per animal, and the number of $\alpha$-motoneurons counted on 10 slices respectively, was analysed.

\section{Results}

\section{Neurological outcome and sciatic functional index (SFI)}

All animals were neurologically intact before sciatic nerve lesion. One day after nerve lesion, signs of a severe motor deficit were found in 17/24 (70.8\%). Signs of a moderate nerve lesion were found in 7/24 (29.2\%) animals in every group. One week after surgery, a marked motor deficit was observed in 14/24 (48.4\%) animals in Group 1 and in 13/24 (44.2\%) animals in Group 2 compared to $16 / 24(66.6 \%)$ animals in Group 3. Two weeks after surgery, 9/12 (75\%) animals in the control group still had signs of a marked motor deficit. In contrast to these findings, $7 / 12(48.4 \%)$ rats treated with
G-CSF after the lesion procedure, and 6/12 (50\%) rats treated with G-CSF both prior to and after surgery, showed a marked motor deficit. At week 6, 4/12 (33.3\%) animals in Group 1 as well as Group 3, and 2/12 (16.7\%) animals in Group 2, showed a marked motor deficit.

At week 6, 4/12 (33.3\%) animals in Group 1 as well as Group 3, and 2/12 (16.7\%) animals in Group 2, showed a marked motor deficit (Fig. 3). Nevertheless, measurements of SFI failed to show statistical significance, although in the long term SFI values of both groups treated with G-CSF improved better than did the SFI values of the control group (Fig. 4).

\section{Motor nerve conduction velocity (NCV) and motor potential amplitude}

Regarding motor NCV as a marker for a damaged myelin sheath, no disparities were observed between the three groups from day 1 to week 2 after nerve lesion. After 4 weeks, nonsignificant improvement of the motor NCV was found in both groups treated with G-CSF compared to the animals treated with G5\%-solution ( $p=0.1$ ). At week 6, Groups 1 and 2 had a significantly better improvement in their motor NCV ratio compared to the control group ( $<$ 0.05) (Fig. 5). Additionally, the development of motor NCV from week 2 to week 6 according to the initial (pre-lesional) NCV value within one group was analysed. Rats receiving G-CSF both pre- and postoperatively improved significantly better referring to the initial NCV $(p<0.05)$ than did those treated with G5\% solution $(p=0.6)$ or with only a preoperative dose of G-CSF $(p=0.2)$.

Besides motor NCV, motor potential amplitude as a marker for loss of sciatic nerve axons was analysed. At week 2, in $6 / 12(50 \%)$ animals in both G-CSF treatment groups, a recovered motor potential amplitude of $>20 \mathrm{~mA}$ was measured. On the contrary, in only $2 / 12(16.6 \%)$ animals in the control group was the amplitude $>20 \mathrm{~mA}$. At week 4 and at week 6 , 6/12 (50\%) rats in Group 1 and 8/12 (66.6\%) rats in Group 2 had motor potential amplitudes of $>20 \mathrm{~mA}$ compared to the control group $(3 / 12 ; 25 \%)$ (Fig. 6). 


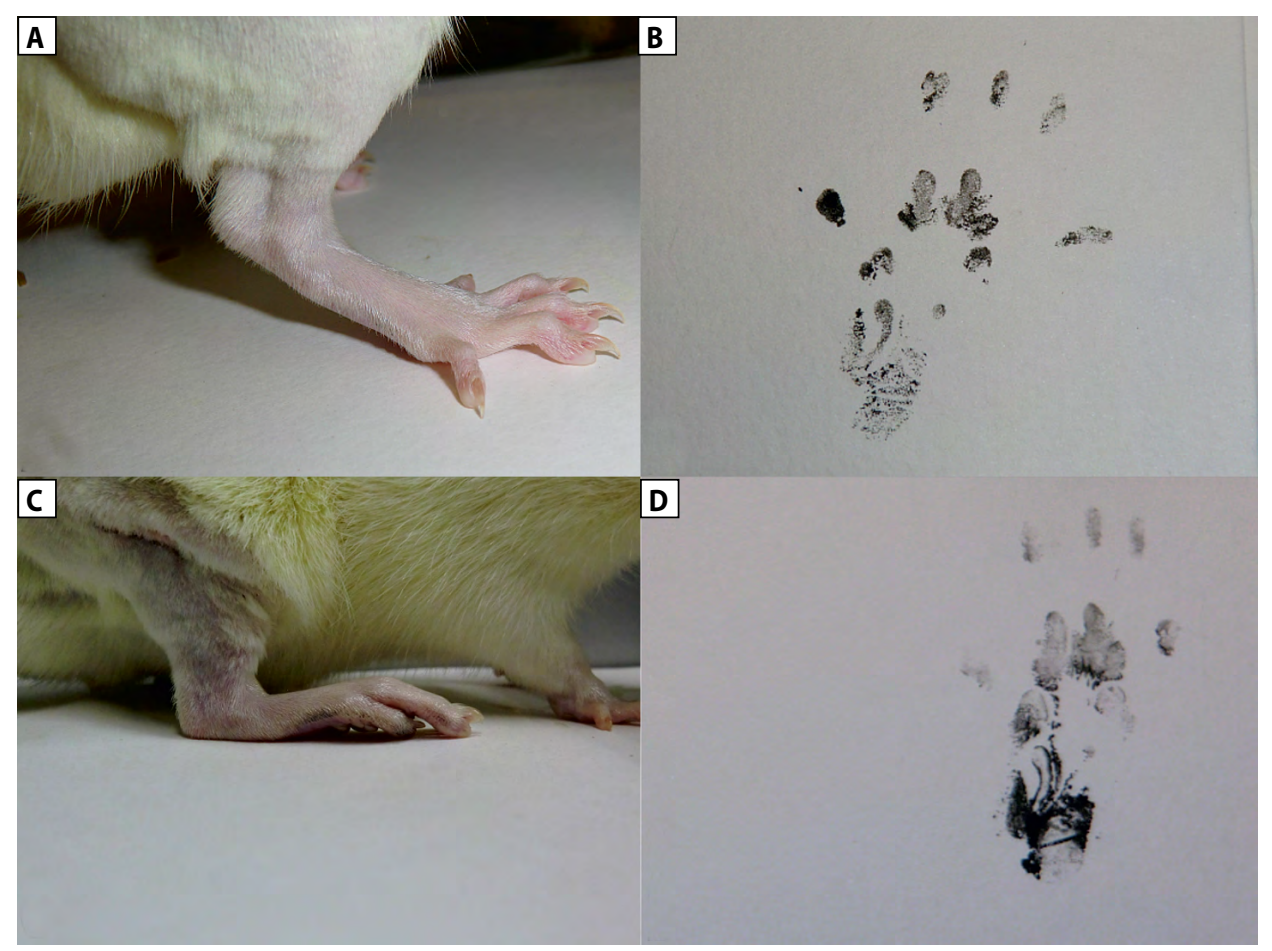

Figure 3. Calculation of sciatic functional index (SFI) was based on a walking track analysis on published values [28]. SFI measurements were performed at weeks 1, 2, 4 and 6. Figures 3A and 3B show a 'physiological' footprint with intact motor function. Figures 3C and 3D show rat footprint after lesion with marked neurological deficit, and correspondingly longer footprint and reduced toe spread. For statistical evaluation, post-injury worsening of SFI was divided into 'intact motor function' = 1 (SFI 0 to -5 ), 'marked neurological deficit' $=2$ (SFI -6 to -50$)$, and 'severe neurological deficit' $=3$ (SFI -51 to -100$)$

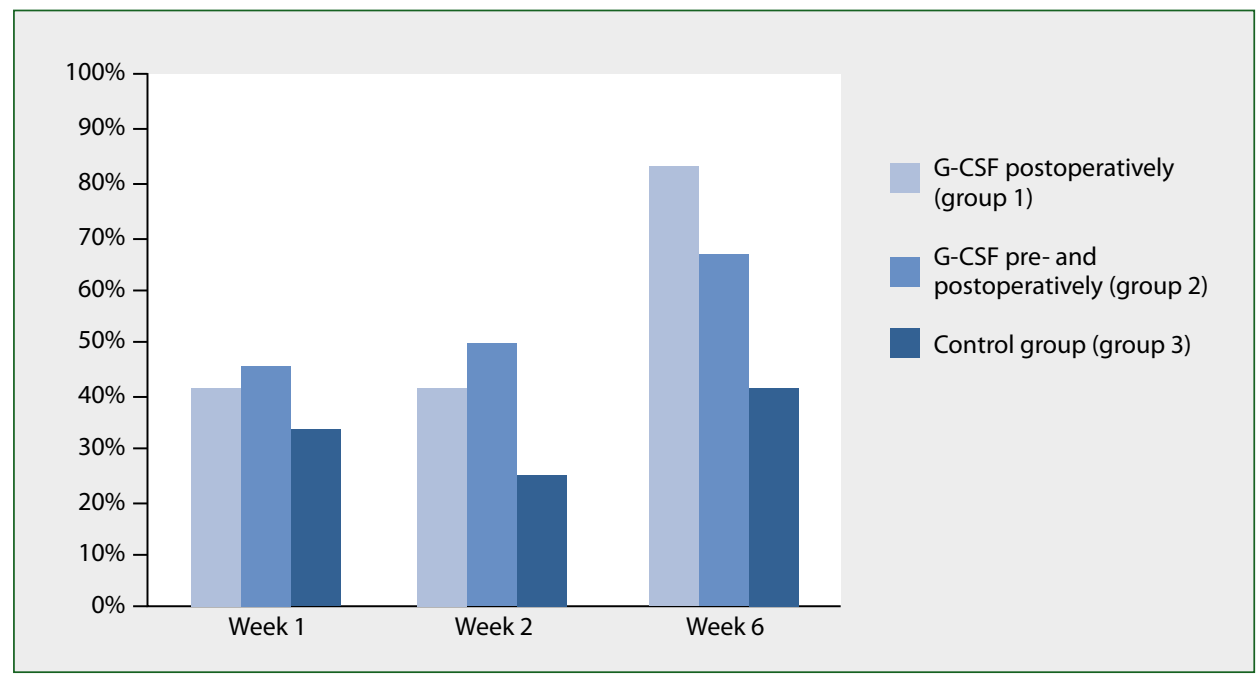

Figure 4. Development of SFI between week 1 and week 6, with proportions of animals without visible motor deficit (SFI 0 to -5$)$ at week 1 , week 2 and week 6 . Compared to control group, motor deficit had recovered in a larger proportion of animals after G-CSF treatment. Although number of animals with recovered motor function was higher after G-CSF treatment at week 2 and week 6 , no statistical difference was observed $(p=0.3$ at week $2 ; p=0.1$ at week 6$)$ 


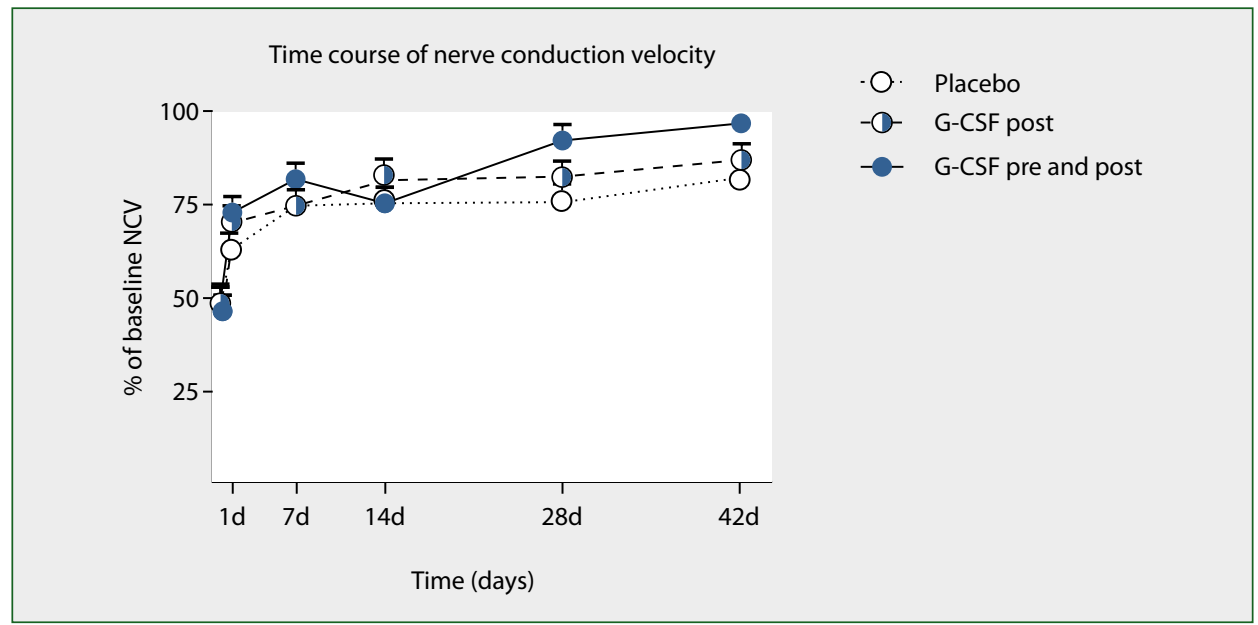

Figure 5. Ratios of preoperative motor NCV directly after lesion procedure, and at day 1, week 1, week 2, week 4, and week 6 . Comparison of ratios shows that motor NCV had better improvement in animals receiving G-CSF, but only in long term after 4 and 6 weeks

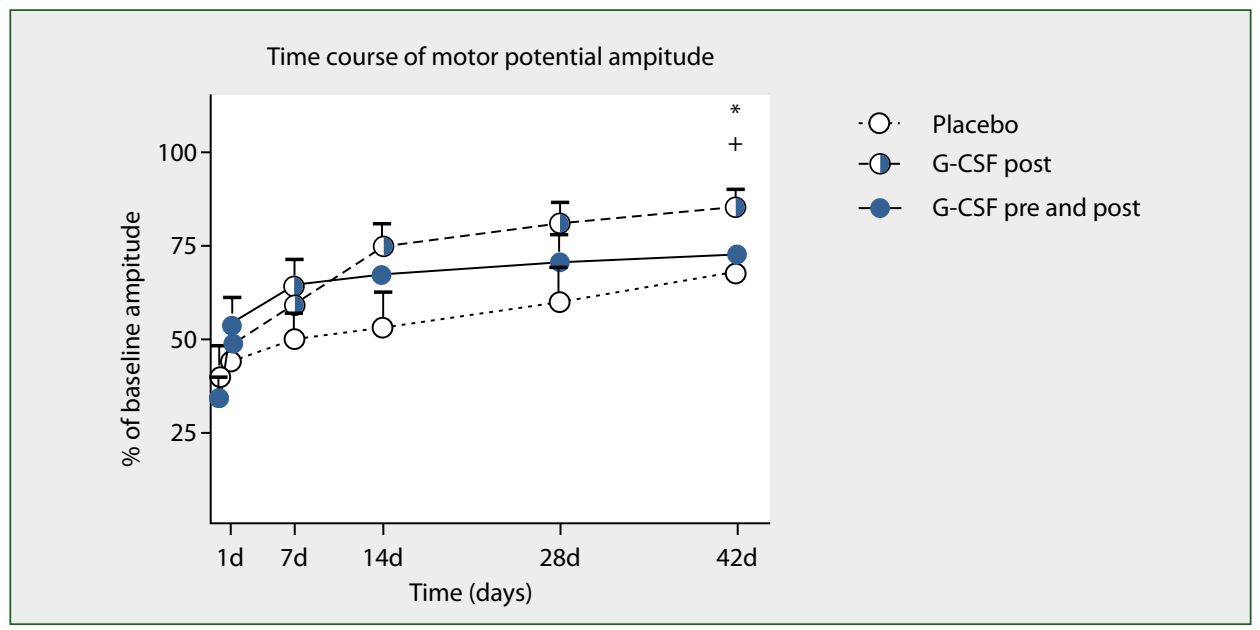

Figure 6. Development of motor potential amplitude after setting of lesion, at day 1 , week 1 , week 2 , week 4 and week 6 . Motor potential amplitude improved quickly between first measurement and week 1 . In general, motor potential amplitude improved more quickly in animals treated with G-CSF

Analysis of changes in motor potential amplitude compared to the baseline amplitude revealed a faster improvement in animals in both G-CSF groups compared to the control group early on from week 1 to week 6 . The recovery of both G-CSF groups was marked at week 1 and week 2 after nerve lesion.

\section{Histological evaluation and morphometric analysis}

Myelin debris and regeneration was evident in all specimens after surgical treatment. In rats sacrificed at week 1 , the total number of nerve fibres and axonal diameters did not differ significantly. On the contrary, six weeks after the surgical procedure, the fibre density and the number of small regenerated axons were significantly higher in animals treated with G-CSF pre- and postoperatively $(\mathrm{p}<0.05)$ (Fig. 7, 8). Due to the considerable increase of small regenerated axons, the fibre density per $\mathrm{mm}^{2}$ and the relative number of large myelinated axons was decreased in these animals. Although animals treated with G-CSF postoperatively showed a slight increase of small regenerated fibres as well, no significant difference in regeneration results was evident in these animals compared to controls.

Counting of $\alpha$-motoneurons (Fig. 9) did reveal a significantly higher number of motoneurons on the lesioned side after G-CSF treatment pre- and postoperatively $(\mathrm{p}<0.05)$ (Fig. 10). Comparison between animals treated pre- and postoperatively and animals that were treated with G-CSF only postoperatively did not show a significant difference in the number of motoneurons. Further, counting of 


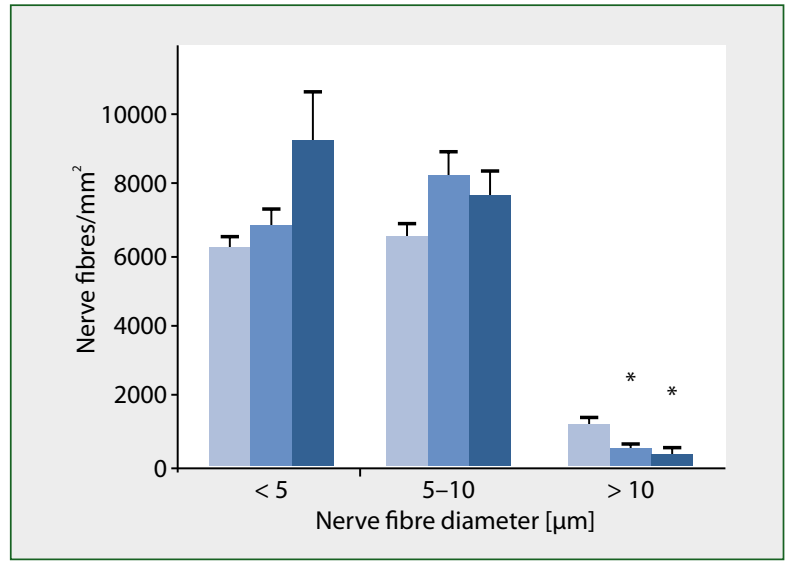

Figure 7. At week 6 , fibre density of sciatic nerve was increased in both groups of animals that had received G-CSF. Additionally, in animals treated with G-CSF pre- and postoperatively, number of small, myelinated nerve fibres of $<5 \mu \mathrm{m}$ in diameter was significantly higher compared to the control group (*; k6 $=$ control group, $\mathrm{p} 6=\mathrm{G}-\mathrm{CSF}$ postoperatively, pp $6=\mathrm{G}-\mathrm{CSF}$ preand postoperatively)

a-motoneurons of the left (intact) side of the spinal cord sections did not reveal a significantly different number between the groups.

\section{Discussion}

Despite numerous research efforts, traumatic or iatrogenic damage to peripheral nerves often results in persistent and/or severe neurological deficits, even where the macro-morphologic nerve structure remains intact. Besides biomechanical or neurophysiological approaches including diverse nerve graft techniques [29-32], several systemic therapeutic agents have been closely investigated, including the administration of neurotrophic factors [33-35]. In recent years, haematopoietic factor G-CSF has been ascribed as having direct protective effects on neurons. The G-CSF receptor and its ligand are expressed in the cortex layers II and V, hippocampus, subventricular zone and in Purkinje-cells [1]. Additionally, G-CSF receptor has been found in deep cerebellar and brain stem nuclei in rodents and humans $[8,36]$. Besides its anti-apoptotic and neuroregenerative properties, G-CSF fosters the formation of vessels after brain ischaemia and improves the recovery of sensorimotor and cognitive functions after stroke, in both experimental models and clinical settings $[1,3,8]$. In contrast to stroke models, functional neurological outcome after sciatic nerve lesion and G-CSF therapy has been rarely investigated [4].

In the spinal cord, G-CSF and its receptor have been detected in a-motoneurons of the ventral horn $[9,10]$. Henriques et al. observed in an animal model of ALS that G-CSF receptor and its ligand are strongly expressed by a-motoneurons [9]. After axotomy of the sciatic nerves in transgenic

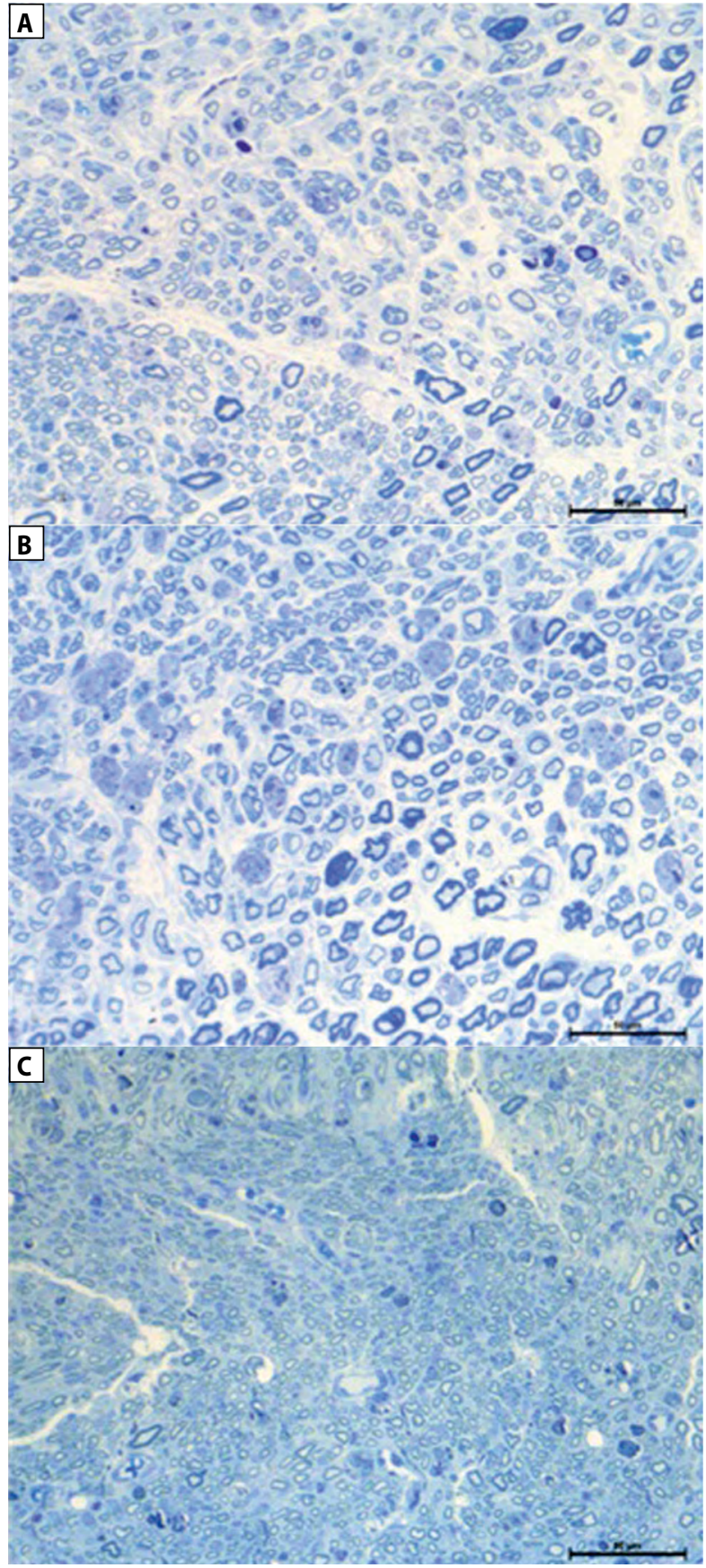

Figure 8. Semi-thin sections of sciatic nerves after 6 weeks of regeneration (methylene blue, scale bars $=50 \mu \mathrm{m}$ ). Control group (Group 3; A), animals treated with G-CSF postoperatively (Group 1; B), and animals treated with G-CSF pre- and postoperatively (Group 2; C). Best regeneration was seen in Group 2, showing a significant increase in number of small, myelinated nerve fibres compared to Group 3

SOD1-mice, a positive influence of G-CSF on motoneuron apoptosis of lumbar $\alpha$-motoneurons with improvement of neuronal survival has been described. Additionally, G-CSF has been shown to preserve the size of motoneurons after axotomy, with increased expression of G-CSF receptor in 


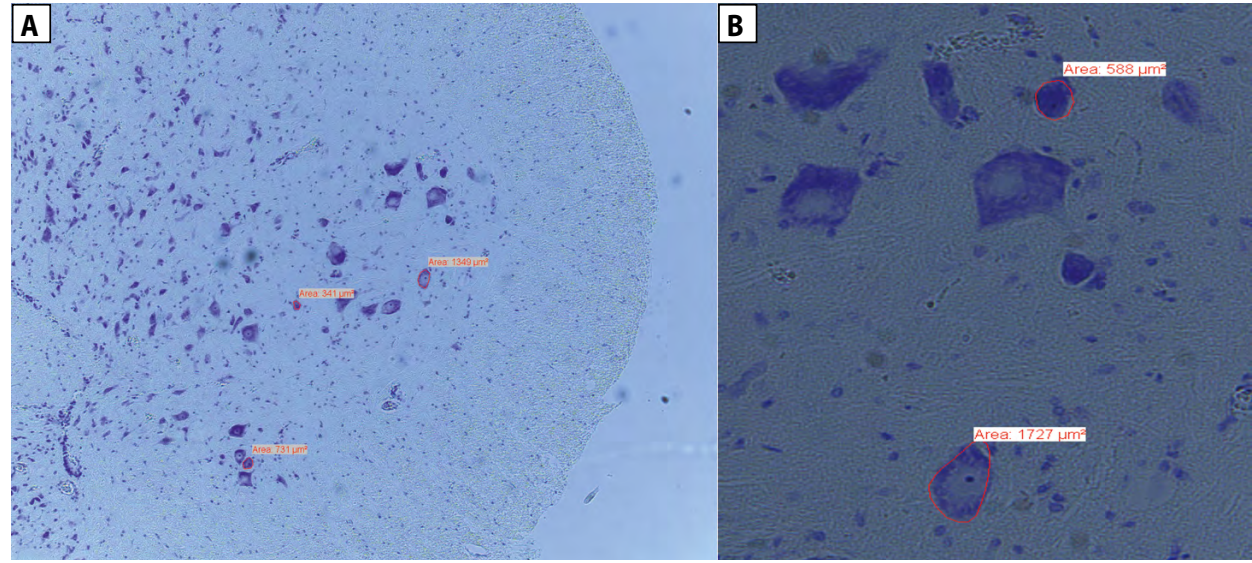

Figure 9. Light microscopic 45-fold enlargement (A) and 250-fold enlargement (B) of a lumbar cord section (HE-staining; SIS AnalySIS software, Olympus). Prior to microscopic analysis, spinal cord sections were fixed in paraffin and cut into $10-\mathrm{mm}$ thick slices. All $\alpha$-motoneurons in laminas 8 and 9 of the ventral horn that were clearly identifiable were counted on both sides. $\alpha$-motoneurons were defined as neurons with a diameter of $\geq 300 \mu \mathrm{m}^{2}$, an intact cell membrane, a clearly definable nuclear membrane with a nucleolus, and clearly definable cytoplasm

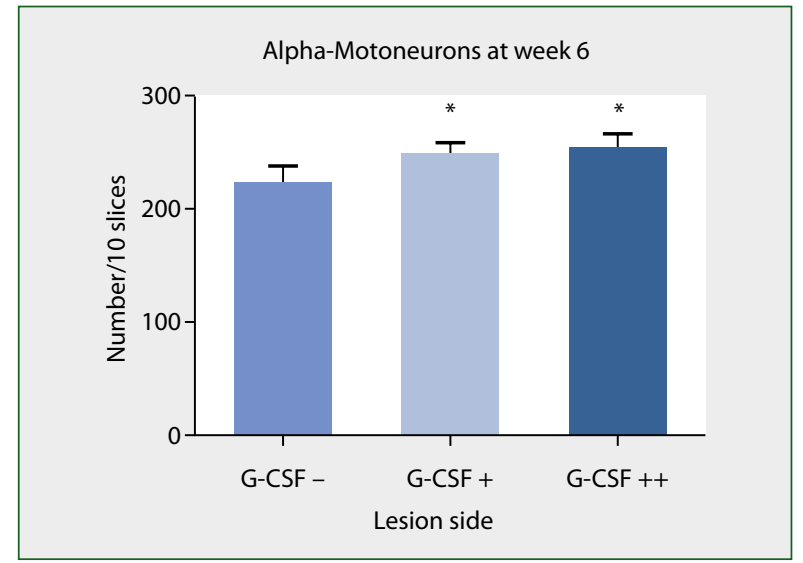

Figure 10. Number of $\alpha$-motoneurons (mean value of 10 consecutive slices of lumbar spinal cord sections) of lesioned side at week 6 . Number of $\alpha$-motoneurons was significantly higher $(* ; p<0.05)$ in animals after G-CSF treatment compared to control group. Comparison between animals that received G-CSF pre- and postoperatively and animals that received G-CSF only postoperatively did not show significant differences

motoneurons at the L4 and L5 levels of the lumbar spine. However, neither the impact of G-CSF on functional impairment nor the number of preserved and regenerated nerve fibres was assessed in that study [9].

In our current study, the potential neuroregenerative effects of G-CSF after reproducible and incomplete peripheral nerve trauma with preserved nerve continuity were investigated. Besides the assessment of histological changes such as the number of $a$-motoneurons or the number and size of sciatic nerve fibres, the main part of our study included the evaluation of changes in motor function and electrophysiological measurement as carried out in clinical settings.
The improvement of motor deficits in animals treated with G-CSF pre- and postoperatively and with G-CSF postoperatively, seemed superior compared to the control group, contradicting the results of Pan et al. [4] who reported no significance after similar treatment. The explanation may lie in the different experimental design, in particular in the different severity of sciatic nerve lesions.

In stroke models, G-CSF promotes a strong anti-apoptotic effect via activation of the PI3K/Akt pathway and to a lesser extent through activation of the STAT3 and ERK5 pathways [37]. To date, the effect of G-CSF on spinal a-motoneurons after lesion of peripheral nerves has not been systematically investigated. The involvement of several pathways including bcl-proteins, AI, IAPs and caspases has been reported [9, 30, 38]. In general, our findings align with the results of Henriques et al. [9] and with results in the treatment of central lesions. Thus, it appears that neuroregenerative effects mediated by G-CSF after peripheral nerve lesion are possible. Although no statistically significant functional benefit could be shown, long-term beneficial effects regarding peripheral nerve regeneration after (incomplete) traumatic nerve lesion might exist.

Regarding the motor NCV ratio and its improvement in the current study, a strong trend towards faster improvement was observed in the animals treated with G-CSF at week 4 and week 6. After six weeks, motor NCV improved significantly in animals that received G-CSF compared to placebo-treated animals. Additionally, the dynamics of recovery in motor potential amplitude (representing the number of functional axons / number of regenerating motor nerve fibres) was more rapid in G-CSF-treated animals compared to those receiving a placebo. The maximal improvement was seen between week 4 and week 6 . This observation aligns with the nerve fibre count surveyed at week 6 . Here, we documented a significantly 
higher number and density of myelinated fibres in animals who received G-CSF. Previous morphological studies have reported that regenerated nerve fibres are smaller, showing a diameter $<5 \mu \mathrm{m}[32,39]$. Thus, in our experiment, the large number of small myelinated fibres might indicate an improved regeneration. Also the $\alpha$-motoneurons count was higher in animals treated with G-CSF.

We conclude that anti-apoptotic effects may lead to an increased survival of $\alpha$-motoneurons and to the initiation/ maintenance of regrowth of their axons i.e. motor fibres.

There are some limitations of the present study. Firstly, the translation of data from an experimental setting to bedside has to be done very carefully and requires additional safety analysis in clinical conditions. Secondly, the design of the present study bears the risk of a certain bias due to a larger deviation of results due to between-subject variation in conditions of electrophysiogical assessment. Finally, calculating statistics in a small cohort carries an increased risk of bias.

On the other hand, the multiple strengths of this study rely on prolonged follow-up of electrophysiological and functional nerve recovery, demonstrating a certain similarity to the clinical routine. To the best of our knowledge, this is the first animal study to evaluate the neuroregenerative potential of G-CSF over a period of up to six weeks, using both functional and electroneurophysiological assessments. Furthermore, the waterjet injury technique offers a high reproducibility of nerve damage with I) maintained nerve continuity, II) a rapid form of injury such as is observed in an acute trauma, and III) the possibility of adjusting the injury severity by altering jet pressure level.

\section{Clinical implications and future directions}

We believe further investigation of nerve regeneration under G-CSF treatment is worth elucidating in clinical settings. To date, no single efficient pharmacological intervention stimulating a peripheral nerve to recover after mechanical damage has been described.

Even if the role of G-CSF in the regeneration of axons and a-motoneurons after such injury is not completely understood, the results of the present study show a structural improvement of nerve regeneration after the application of G-CSF and acceleration of its functional recovery.

In cases of scheduled neurosurgical procedures on peripheral nerves with an (expected) traumatic impact on the nerve due to prolonged or intense manipulation, G-CSF could be administered prior to surgery. Thus, additional studies investigating the neuroprotective and neuroregenerative mechanisms of G-CSF after peripheral nerve lesions are important. G-CSF has been administered in humans for many years to treat neutropenia, and might be a promising agent after peripheral nerve lesion. However, better understanding of the potential effects of G-CSF in terms of neuroregenerative mechanisms, G-CSF dose, and form of G-CSF administration is of the utmost importance. In the long term, studies investigating G-CSF and its potential to preserve the peripheral nerve or support its regeneration under clinical circumstances may well be worth initiating.

Author disclosure statement: J. Oertel is consultant to Karl Storz Company. J. Oertel has received grants from Erbe Company. These companies were not involved in the experimental set up, work or analysis of the present study. Other than this, all authors declare that they have no competing financial interest. This study was financially supported by the non-profit foundation Else-Kröner-Fresenius-Stiftung.

\section{References}

1. Schneider A, Krüger C, Steigleder T, et al. The hematopoietic factor G-CSF is a neuronal ligand that counteracts programmed cell death and drives neurogenesis. J Clin Invest. 2005; 115(8): 2083-2098, doi: 10.1172/JCl23559, indexed in Pubmed: 16007267.

2. Strecker JK, Sevimli S, Schilling M, et al. Effects of G-CSF treatment on neutrophil mobilization and neurological outcome after transient focal ischemia. Exp Neurol. 2010; 222(1): 108-113, doi: 10.1016/j. expneurol.2009.12.012, indexed in Pubmed: 20026112.

3. Schäbitz WR, Schneider A. New targets for established proteins: exploring G-CSF for the treatment of stroke. Trends Pharmacol Sci. 2007; 28(4): 157-161, doi: 10.1016/j.tips.2007.02.007, indexed in Pubmed: 17350693.

4. Pan HC, Wu HT, Cheng FC, et al. Potentiation of angiogenesis and regeneration by G-CSF after sciatic nerve crush injury. Biochem Biophys Res Commun. 2009; 382(1): 177-182, doi: 10.1016/j. bbrc.2009.03.003, indexed in Pubmed: 19275877.

5. Diederich K, Quennet V, Bauer H, et al. Successful regeneration after experimental stroke by granulocyte-colony stimulating factor is not further enhanced by constraint-induced movement therapy either in concurrent or in sequential combination therapy. Stroke. 2012; 43(1): 185-192, doi: 10.1161/STROKEAHA.111.622159, indexed in Pubmed: 22020031.

6. Gibson CL, Bath PMW, Murphy SP. G-CSF reduces infarct volume and improves functional outcome after transient focal cerebral ischemia in mice. J Cereb Blood Flow Metab. 2005; 25(4): 431-439, doi: 10.1038/sj.jcbfm.9600033, indexed in Pubmed: 15660101.

7. Lee ST, Chu K, Jung KH, et al. Granulocyte colony-stimulating factor enhances angiogenesis after focal cerebral ischemia. Brain Res. 2005; 1058(1-2): 120-128, doi: 10.1016/j.brainres.2005.07.076, indexed in Pubmed: 16150422.

8. Schäbitz WR, Schneider A. Developing granulocyte-colony stimulating factor for the treatment of stroke: current status of clinical trials. Stroke. 2006; 37(7): 1654; author reply 1655, doi: 10.1161/01. STR.0000227299.62106.0e, indexed in Pubmed: 16728676.

9. Henriques A, Pitzer C, Dupuis L, et al. G-CSF protects motoneurons against axotomy-induced apoptotic death in neonatal mice. BMC Neurosci. 2010; 11: 25, doi: 10.1186/1471-2202-11-25, indexed in Pubmed: 20178614.

10. Pitzer C, Krüger C, Plaas C, et al. Granulocyte-colony stimulating factor improves outcome in a mouse model of amyotrophic lateral sclerosis. Brain. 2008; 131(Pt 12): 3335-3347, doi: 10.1093/brain/ awn243, indexed in Pubmed: 18835867.

11. Oertel J, Gaab MR, Knapp A, et al. Water jet dissection in neurosurgery: experimental results in the porcine cadaveric brain. Neurosurgery. 
2003; 52(1): 153-9; discussion 159, doi: 10.1097/00006123200301000-00020, indexed in Pubmed: 12493113.

12. Oertel J, Gaab MR, Piek J. Waterjet resection of brain metastases first clinical results with 10 patients. Eur J Surg Oncol. 2003; 29(4): 407-414, doi: 10.1053/ejso.2002.1428, indexed in Pubmed: 12711300.

13. Oertel J, Gaab MR, Pillich DT, et al. Comparison of waterjet dissection and ultrasonic aspiration: an in vivo study in the rabbit brain. J Neurosurg. 2004; 100(3): 498-504, doi: 10.3171/ jns.2004.100.3.0498, indexed in Pubmed: 15035286.

14. Oertel J, Gaab MR, Runge U, et al. Waterjet dissection versus ultrasonic aspiration in epilepsy surgery. Neurosurgery. 2005; 56(1 Suppl): $142-$ 6; discussion 142, doi: 10.1227/01.neu.0000144316.87764.13, indexed in Pubmed: 15799802.

15. Oertel J, Gaab MR, Warzok R, et al. Waterjet dissection in the brain: review of the experimental and clinical data with special reference to meningioma surgery. Neurosurg Rev. 2003; 26(3): 168-174, doi: 10.1007/s10143-002-0244-7, indexed in Pubmed: 12845544.

16. Piek J, Oertel J, Gaab MR. Waterjet dissection in neurosurgical procedures: clinical results in 35 patients. J Neurosurg. 2002; 96(4): 690-696, doi: 10.3171/jns.2002.96.4.0690, indexed in Pubmed: 11990809.

17. Piek J, Wille C, Warzok R, et al. Waterjet dissection of the brain: experimental and first clinical results. Technical note. J Neurosurg. 1998; 89(5): 861-864, doi: 10.3171/jns.1998.89.5.0861, indexed in Pubmed: 9817429.

18. Keiner D, Gaab MR, Backhaus V, et al. Water jet dissection in neurosurgery: an update after 208 procedures with special reference to surgical technique and complications. Neurosurgery. 2010; 67(2 Suppl Operative): 342-354, doi: 10.1227/NEU.0b013e3181f743bb, indexed in Pubmed: 21099557.

19. Tschan C, Gaab MR, Krauss JK, et al. Waterjet dissection of the vestibulocochlear nerve: an experimental study. J Neurosurg. 2009; 110(4): 656-661, doi: 10.3171/2008.5.17561, indexed in Pubmed: 18834266.

20. Tschan CA, Keiner D, Müller HD, et al. Waterjet dissection of peripheral nerves: an experimental study of the sciatic nerve of rats. Neurosurgery. 2010; 67(2 Suppl Operative): 368-376, doi: 10.1227/ NEU.0b013e3181f9b0c8, indexed in Pubmed: 21099560.

21. Dinh $P$, Hazel A, Palispis W, et al. Functional assessment after sciatic nerve injury in a rat model. Microsurgery. 2009; 29(8): 644-649, doi: 10.1002/micr.20685, indexed in Pubmed: 19653327.

22. Kalender AM, Dogan A, Bakan V, et al. Effect of Zofenopril on regeneration of sciatic nerve crush injury in a rat model. J Brachial Plex Peripher Nerve Inj. 2009; 4: 6, doi: 10.1186/1749-7221-4-6, indexed in Pubmed: 19508704.

23. Yan JG, Matloub HS, Yan Y, et al. The correlation between calcium absorption and electrophysiological recovery in crushed rat peripheral nerves. Microsurgery. 2010; 30(2): 138-145, doi: 10.1002/ micr.20709, indexed in Pubmed: 19790186.

24. Kouyoumdjian JA. Peripheral nerve injuries: a retrospective survey of 456 cases. Muscle Nerve. 2006; 34(6): 785-788, doi: 10.1002/ mus.20624, indexed in Pubmed: 16881066.

25. Eser $\mathrm{F}$, Aktekin LA, Bodur $\mathrm{H}$, et al. Etiological factors of traumatic peripheral nerve injuries. Neurol India. 2009; 57(4): 434-437, doi: 10.4103/0028-3886.55614, indexed in Pubmed: 19770544.

26. Kretschmer T, Antoniadis G, Braun V, et al. Evaluation of iatrogenic lesions in 722 surgically treated cases of peripheral nerve trauma. J Neurosurg. 2001; 94(6): 905-912, doi: 10.3171/ jns.2001.94.6.0905, indexed in Pubmed: 11409518.

27. Wee AS, Truitt NR, Smith LD. Type and frequency of peripheral nerve injuries encountered in a clinical neurophysiology laboratory. Journal of the Mississippi State Medical Association. 2006; 47: 67-71.

28. de Medinaceli L, Freed WJ, Wyatt RJ. An index of the functional condition of rat sciatic nerve based on measurements made from walking tracks. Exp Neurol. 1982; 77(3): 634-643, doi: 10.1016/00144886(82)90234-5, indexed in Pubmed: 7117467.

29. McGrath AM, Brohlin M, Kingham PJ, et al. Fibrin conduit supplemented with human mesenchymal stem cells and immunosuppressive treatment enhances regeneration after peripheral nerve injury. Neurosci Lett. 2012; 516(2): 171-176, doi: 10.1016/j.neulet.2012.03.041, indexed in Pubmed: 22465323.

30. Imaizumi K, Benito A, Kiryu-Seo S, et al. Critical role for DP5/Harakiri, a Bcl-2 homology domain 3-only Bcl-2 family member, in axotomy-induced neuronal cell death. J Neurosci. 2004; 24(15): 3721-3725, doi: 10.1523/JNEUROSCI.5101-03.2004, indexed in Pubmed: 15084651.

31. Belkas JS, Munro CA, Shoichet MS, et al. Long-term in vivo biomechanical properties and biocompatibility of poly(2-hydroxyethyl methacrylate-co-methyl methacrylate) nerve conduits. Biomaterials. 2005; 26(14): 1741-1749, doi: 10.1016/j.biomaterials.2004.05.031, indexed in Pubmed: 15576148.

32. Kemp SWP, Webb AA, Dhaliwal S, et al. Dose and duration of nerve growth factor (NGF) administration determine the extent of behavioral recovery following peripheral nerve injury in the rat. Exp Neurol. 2011; 229(2): 460-470, doi: 10.1016/j.expneurol.2011.03.017, indexed in Pubmed: 21458449.

33. Alrashdan MS, Sung MA, Kwon YK, et al. Effects of combining electrical stimulation with BDNF gene transfer on the regeneration of crushed rat sciatic nerve. Acta Neurochir (Wien). 2011; 153(10): 2021-2029, doi: 10.1007/s00701-011-1054-x, indexed in Pubmed: 21656118.

34. Devesa P, Gelabert M, Gonźlez-Mosquera T, et al. Growth hormone treatment enhances the functional recovery of sciatic nerves after transection and repair. Muscle Nerve. 2012; 45(3): 385-392, doi: 10.1002/mus.22303, indexed in Pubmed: 22334173.

35. Haastert-Talini $\mathrm{K}$, Schmitte R, Korte N, et al. Electrical stimulation accelerates axonal and functional peripheral nerve regeneration across long gaps. J Neurotrauma. 2011; 28(4): 661-674, doi: 10.1089/ neu.2010.1637, indexed in Pubmed: 21265597.

36. Ridwan S, Bauer H, Frauenknecht $\mathrm{K}$, et al. Distribution of granulocyte-monocyte colony-stimulating factor and its receptor $\alpha$-subunit in the adult human brain with specific reference to Alzheimer's disease. J Neural Transm (Vienna). 2012; 119(11): 1389-1406, doi: 10.1007/s00702-012-0794-y, indexed in Pubmed: 22430742.

37. Minnerup J, Sevimli S, Schäbitz WR. Granulocyte-colony stimulating factor for stroke treatment: mechanisms of action and efficacy in preclinical studies. Exp Transl Stroke Med. 2009; 1: 2, doi: 10.1186/2040-7378-1-2, indexed in Pubmed: 20142989.

38. Perrelet $D$, Ferri A, MacKenzie AE, et al. IAP family proteins delay motoneuron cell death in vivo. Eur J Neurosci. 2000; 12(6): 2059-2067, doi: 10.1046/j.1460-9568.2000.00098.x, indexed in Pubmed: 10886345.

39. Lee AC, Yu VM, Lowe JB, et al. Controlled release of nerve growth factor enhances sciatic nerve regeneration. Exp Neurol. 2003; 184(1): 295-303, doi: 10.1016/s0014-4886(03)00258-9, indexed in Pubmed: 14637100. 